\title{
Lung Ultrasonography May Provide an Indirect Estimation of Lung Porosity and Airspace Geometry
}

\author{
Gino Soldati $^{\mathrm{a}}$ Andrea Smargiassi $^{\mathrm{b}}$ Riccardo Inchingolo $^{\mathrm{b}}$ Sara Sher $^{\mathrm{c}}$ \\ Rosanna Nenna $^{d}$ Salvatore Valente ${ }^{b}$ Cosimo Damiano Inchingolo ${ }^{d}$ \\ Giuseppe Maria Corbo ${ }^{b}$

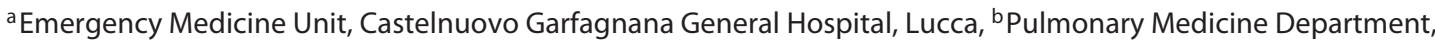 \\ University Hospital 'A. Gemelli', Rome, 'Anesthesia and Intensive Care Unit, Niguarda Cà Granda Hospital, Milan, and \\ dSurgical Pathology Department, 'L. Bonomo' General Hospital, Andria, Italy
}

\section{Key Words}

Chest ultrasound $\cdot$ Lung density $\cdot$ Lung porosity .

Sonographic interstitial syndrome $\cdot$ B-lines

\begin{abstract}
Background: Echographic vertical artifacts (B-lines) in chest ultrasonography have often been associated with pathological patterns. A scientifically sound explanation of these artifacts has not yet been proposed. Objectives: The 'spongy' nature of the lung in its liquid and solid components and the changes that take place in peripheral airspace (PAS) geometry might be the key point to understanding these phenomena. Methods: Six excised right rabbit lungs were obtained. Each lung underwent direct ultrasound evaluation in two different conditions: at complete tissue elastic recoil volume and at pulmonary expansion volume achieved by applying a constant positive pressure of $12 \mathrm{~cm}$ $\mathrm{H}_{2} \mathrm{O}$. Lung volumes and densities were reported in both conditions. Histological examination was performed on three naturally collapsed lungs and on three lungs under positive pressure inflation after having been fixed in forma-
\end{abstract}

lin solution. Results: Mean volumes of naturally collapsed lungs and fixed expanded lungs were $11.2 \pm 0.36$ and 44.83 $\pm 3.03 \mathrm{ml}$, respectively. Mean densities were $0.622 \pm 0.016$ and $0.155 \pm 0.007 \mathrm{~g} / \mathrm{ml}$, respectively. Ultrasound evaluation of collapsed lungs showed dense vertical artifacts and a 'white lung' pattern, while the evaluation of expanded lungs showed hyperechoic line and horizontal artifacts of reflection. Histological evaluation showed a different PAS geometry in collapsed lungs caused by alveolar size reduction and shape changes with unfolded and closed units modifying the peripheral porosity of the frothy nature of the lung. Conclusions: Airspace geometry, frothy nature and porosity are the determinants of the different behavior of ultrasound interacting with the subpleural lung parenchyma. Chest ultrasound may thus be interpreted as an indirect 'estimator' of lung porosity.

(c) 2014 S. Karger AG, Basel

G.S., A.S. and R.I. contributed equally to this work.

This work was accepted as an abstract at the ERS Congress 2014 in Munich, Germany.

\section{KARGER}

E-Mail karger@karger.com www.karger.com/res
(C) 2014 S. Karger AG, Basel

$0025-7931 / 14 / 0886-0458 \$ 39.50 / 0$
Andrea Smargiassi, MD

Pulmonary Medicine Departmen

Università Cattolica del Sacro Cuore

Largo Gemelli 8, IT-00168 Rome (Italy)

E-Mail smargiassi.a@gmail.com 


\section{Introduction}

Lung ultrasonography is obtaining acceptance as a diagnostic modality. Its role in different clinical settings has already been shown [1-7]. The difficulties in making this technique widely accepted are based on the ability of ultrasound to cross tissues and make a morphological analysis of anatomic structures. It is well known that inflated lungs contain air and that the ultrasound beam does not penetrate the pleural plane because of the large difference in acoustic impedance between chest wall tissues and the lungs [8]. A strong hyperechogenic interface, the pleural line, represents the ultimate border of 'morphological analysis' underneath which a series of mirror phenomena take place due to reverberating interfaces. These horizontal reverberating artifacts, named A-lines, have been associated to the normal aerated lung echographic assessment $[1,4]$.

An anatomical analysis of lung parenchyma is only possible when the lung is completely deflated and consolidated, as occurs in atelectasis or pneumonia (lung hepatization) [9-12]. However, in the ultrasound assessment of not completely consolidated pathological lungs, as in cardiogenic or noncardiogenic pulmonary edema, the appearance of vertical artifactual phenomena has been described (B-lines or 'white lung') and identifies the sonographic interstitial syndrome $[2,4,13]$.

To date, qualitative hypotheses have only been proposed regarding the origin of these vertical artifacts. Resonance phenomena of bullous tetrahedral structures and ring-down artifacts $[14,15]$, reverberating interlobular septa [16] and hemorrhagic iatrogenic damage [17] appear to be only partially satisfactory explanations.

Thanks to previous studies of bioacoustics, we have understood that the sound power transmission coefficient is directly related to lung density $[18,19]$. Previous investigations have already demonstrated that vertical artifacts can be reproduced using a wet foam of polyurethane, depending on its porosity [20].

The 'spongy' nature of the lung in its full and empty components might be the key point to understanding these echographic artifactual phenomena in the case of changing superficial density and porosity in terms of peripheral airspace (PAS) geometry. The aim of this study is to compare histological features of healthy lung parenchyma in two different conditions and observe the different echographic patterns created in order to understand their nature and try to find a unifying theory on the genesis of vertical artifacts in chest ultrasonography.

Lung Ultrasonography and Estimation of Porosity and Airspace Geometry

\section{Materials and Methods}

This study was approved by the Institutional Care and Animal Use Committee (IACUC) of the Catholic University of the Sacred Heart in Rome, and it was performed according to the Helsinki Declaration. Six right lungs with their intact right main bronchi were obtained from six commercially available European white rabbits (Oryctolagus cuniculus, about 3 months of age, mean weight 3,045 $\pm 70 \mathrm{~g}$ ). Under sodium pentobarbital anesthesia, the animals were sacrificed through carotid cut and exsanguination, in agreement with Italian laws regarding the use of animals in experiments. An incision was made through the right fourth intercostal space, the right main bronchus with pulmonary hilar vessels was cut and the right lung with its main bronchus removed. Specimens were processed within $2 \mathrm{~h}$ of thoracotomy.

The net weights of the lungs were determined using an electronic precision balance (JAY 1003; Nanjing Oxy Technology \& Trading Co., Nanjing, China) with a reading accuracy of $0.01 \mathrm{~g}$.

Hemithorax mean resting chest wall volume measured by a postmortem thoracic cast for a 3-kg rabbit [21] was used as a comparison to estimate lung volumes. The intact lungs, connected via an open bronchial polyethylene catheter $(40 \mathrm{~mm}$ in length and $3 \mathrm{~mm}$ in internal diameter) to a custom electronic pressure controller maintained at $0 \mathrm{~cm} \mathrm{H}_{2} \mathrm{O}$, were placed in a custom-made chamber filled with phosphate buffer solution in order to obtain their natural collapse (complete tissue elastic recoil).

Ultrasound lung evaluation was performed directly on the anterior, lateral and posterior surfaces of each collapsed lung using a 5- to $10-\mathrm{mm}$ thick protected specimen brush covering the specimen as acoustic coupling media (Toshiba Aplio XV US machine equipped with a 4-cm, 9-MHz linear probe, Toshiba PLT 805 AT). No harmonic imaging was used.

All lungs underwent a controlled inflation achieved by applying a constant positive pressure through the polyethylene catheter previously inserted in the bronchial lumen. To obtain constant expansion, compensate for leakage and prevent pulmonary deflation, a positive pressure of $12 \mathrm{~cm} \mathrm{H}_{2} \mathrm{O}$ was produced by continuous manometer-controlled air flow. Representative ultrasound findings (3 longitudinal echographic scans) from anterior, lateral and posterior sides of each inflated specimen were stored on the machine's hard disk for subsequent evaluation.

Lung volumes in the condition of natural collapse and inflation were measured by water displacement. The lungs were submerged in a graded recipient of known volume of distilled water and the displaced meniscus on a graduated scale (reading accuracy $0.1 \mathrm{ml}$ ) was measured. Lung densities were calculated by the ratio between weight and volume of each lung.

\section{Histologic Examination and Image Processing}

After image acquisition, three random lungs were deflated to complete tissue elastic recoil volume and fixed in 10\% neutral formalin solution for $48 \mathrm{~h}$. In the other three expanded lungs instead, the vascular bed was first washed out with saline solution then fixation was carried out through the pulmonary artery. Adjunctive formalin solution (10\%) was nebulized through the airways [22] by connecting the nebulizer to the pressure delivery system so as to fix the distal airway spaces in the controlled expanded state for $24 \mathrm{~h}$. Lungs were subsequently postfixed by immersion in $10 \%$ formalin for 10 days. 
Table 1. Physical characteristics of rabbits and lungs

\begin{tabular}{|c|c|c|c|c|c|c|}
\hline \multirow[t]{2}{*}{ Rabbit } & \multirow{2}{*}{$\begin{array}{l}\text { Body weight, } \\
\mathrm{g}\end{array}$} & \multicolumn{2}{|c|}{ Lung volume, $\mathrm{ml}$} & \multirow{2}{*}{$\begin{array}{l}\text { Lung weight, } \\
\mathrm{g}\end{array}$} & \multicolumn{2}{|c|}{ Lung density, g/ml } \\
\hline & & deflated & inflated & & deflated & inflated \\
\hline 1 & 3,120 & 11 & 44 & 7.06 & 0.642 & 0.157 \\
\hline 2 & 2,970 & 10.9 & 41 & 6.68 & 0.613 & 0.163 \\
\hline 3 & 2,950 & 11 & 42 & 6.90 & 0.627 & 0.164 \\
\hline 4 & 3,050 & 11.2 & 46 & 7.08 & 0.632 & 0.154 \\
\hline 5 & 3,100 & 11.9 & 49 & 7.10 & 0.596 & 0.145 \\
\hline 6 & 3,080 & 11.3 & 47 & 7.08 & 0.626 & 0.151 \\
\hline Mean \pm SD & $3,045 \pm 70.07$ & $11.2 \pm 0.36$ & $44.83 \pm 3.03$ & $6.98 \pm 0.16$ & $0.622 \pm 0.016$ & $0.155 \pm 0.007$ \\
\hline
\end{tabular}

Expanded and collapsed lungs were longitudinally cut into $0.5-\mathrm{cm}$-thick sections from the apex to the base through the hilum. Three slices per lung were randomly chosen and embedded in paraffin. Four-micrometer cuts were made and stained with hematoxylin and eosin (HE). Of the remaining slices, one slice per lung was chosen at random and stained with Mallory trichrome (MT). Image capture $(40 \times)$ was performed according to Escolar et al. [23]. From each lung a slice was chosen and divided into 6 subpleural zones, from which 3 were chosen at random. Within each of these, a histological field was also randomly chosen. Fifty-four histological fields (HE) were evaluated for PAS morphometry and 18 fields for interstitial pathology (MT). Finally, three random histological fields $(40 \times)$ of expanded lungs and three random histological fields $(40 \times)$ of collapsed lungs were captured and stored as JPEG 8-bit gray images at a size of $400 \times 250$ pixels for subsequent digital analysis.

Microscopic study, image digitalization and histologic analysis were performed using a Leica DM3000 microscope equipped with Leica Application Suite software (version 3.5.0; Leica Microsystems CMS, Wetzlar, Germany). Image analysis and quantification was performed using Image J (National Institutes of Health, Bethesda, Md., USA). Two pathologists (R.N. and C.D.I.) independently evaluated samples by optical microscopy, and every histological definition required their full concordance.

The microscopic appearance of PAS (acinar and lobular units) and septal tissues was analyzed in both sets of lungs. The interstitial tissue, alveolar epithelium and alveolar lumens were also evaluated for pathologic signs. A simplified planimetric analysis was conducted performing a quantitative estimate (alveolar cords and PAS areas) on all random microscopic fields. The alveolar cord was measured as the distance that exists between two walls of a single PAS, expressed in micrometers, and the area of the PAS was considered as the surface area of a terminal lung unit expressed in micrometers squared.

Six random 40× microscopic HE-stained fields (8-bit JPEG images from three expanded and three collapsed lungs) were digitally transformed in black and white in order to separate the regions corresponding to interstice (black) from the regions corresponding to air spaces (white), considered as background. For each image 15 random $50 \times 50$ pixel fields were chosen and analyzed to quantify the percentage of black area corresponding to the parenchymatous tissue portion. Data were expressed as means and standard deviation, $t$ test was used for comparing paired groups and the statistical significance was considered with $\mathrm{p}<0.05$.

\section{Results}

The weights of the six isolated right lungs ranged between 6.68 and $7.1 \mathrm{~g}$ (mean $6.98 \pm 0.16 \mathrm{~g}$ ). The volumes estimated at complete tissue elastic recoil were between 10.9 and $11.9 \mathrm{ml}$ (mean $11.2 \pm 0.36 \mathrm{ml}$ ). The volumes estimated during positive pressure expansion ranged between 41 and $49 \mathrm{ml}$ (mean volume $44.83 \pm 3.03 \mathrm{ml}$ ). The physical characteristics of the animals and organs in both conditions are reported in table 1 .

It can be estimated [21] that a volume of $45 \mathrm{ml}$ corresponds to about $40 \%$ of the hemithorax mean resting chest wall volume. Schibler and Henning [24] previously reported the functional residual capacity of rabbits during mechanical ventilation (at PEEP 0) to be close to 15 $\mathrm{ml} / \mathrm{kg}$ (about $45 \mathrm{ml}$ for $3 \mathrm{~kg}$ of weight). Moreover, for mammals, Stahl [25] considers as respiratory dimensional constants values near $7.5 \mathrm{ml} / \mathrm{kg}$ for tidal volume, $24 \mathrm{ml} /$ $\mathrm{kg}$ for functional residual capacity and $57 \mathrm{ml} / \mathrm{kg}$ for vital capacity. Thus, we can conclude that a volume of $45 \mathrm{ml}$, referring to a single right lung, corresponds to approximately $200 \%$ of functional residual capacity or, in other words, to a quantity within the middle third of the inspiratory reserve volume. In all three lungs, ultrasound evaluation at a condition of natural isobaric collapse (within $2 \mathrm{~h}$ from chest cage removal), showed a condition of dense vertical artifacts (B-lines) [4] to white lung (consisting in a completely white echographic field with or without merged B-lines and with no horizontal reverberation; fig. 1).

Ultrasound evaluation of the expanded lungs showed only a hyperechoic line, visually corresponding to what we know as the 'pleural line' in human chest ultrasound [4], which was interrupted only in areas of not fully macroscopically expandable lung. This line was continuous and regular, without any evidence of artifactual 
Fig. 1. Ultrasound assessment of naturally collapsed isolated rabbit right lungs. A white lung pattern is clearly shown.
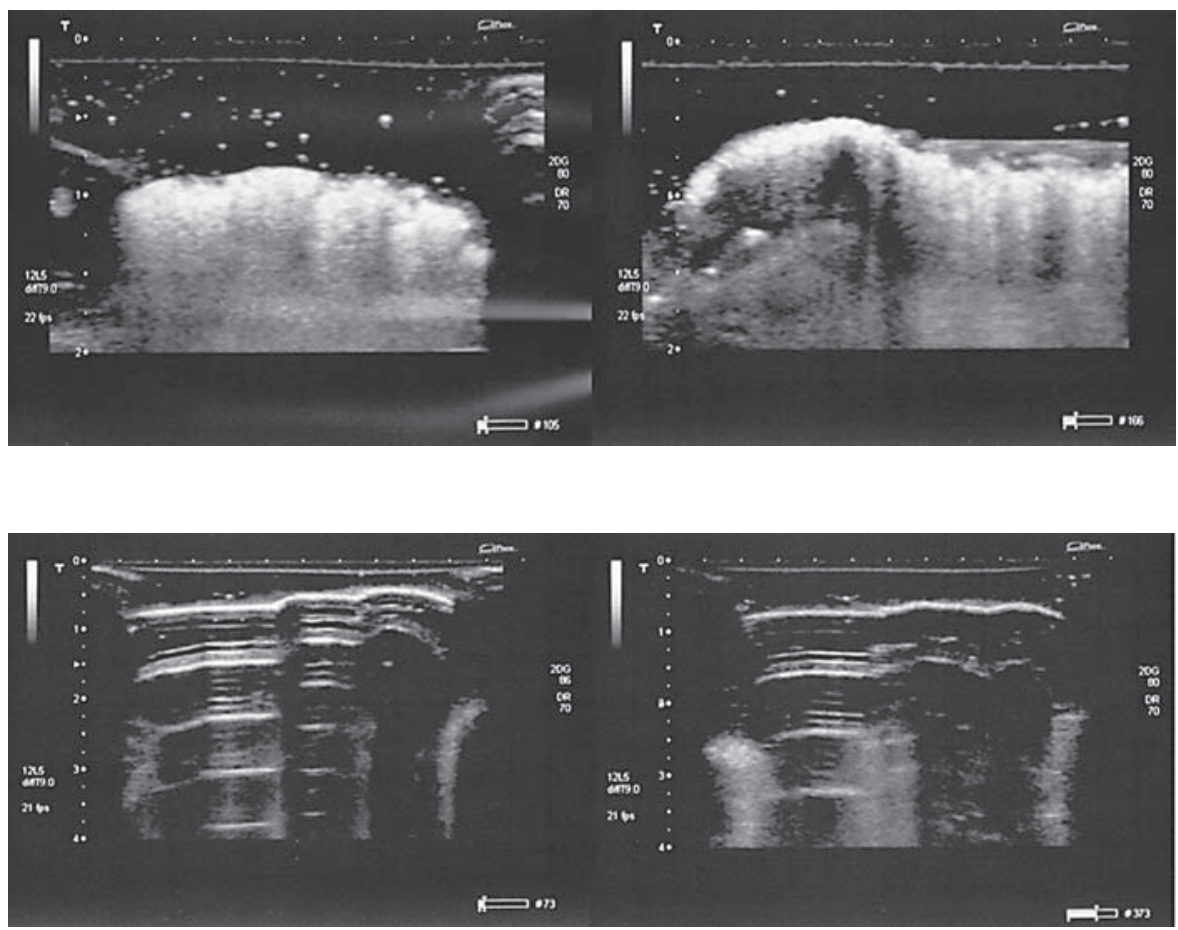

Fig. 2. Ultrasound assessment of inflated isolated rabbit right lungs. A pattern of horizontal artifacts of reverberation is shown.
The mean alveolar cord was $66.6 \pm 17.1 \mu \mathrm{m}$ and the mean peripheral surface area was $2,573.3 \pm 2,123.9 \mu \mathrm{m}^{2}$ (fig. 4).

The ratio (\%) of subpleural parenchymatous tissue calculated by Image J was, on average, $33.4 \pm 10.4$ in inflated lungs, and $69.3 \pm 14.2$ in collapsed lungs. Concordance between the two pathologists was complete. Differences between mean diameters, mean PAS and parenchymal tissue ratio between collapsed and expanded alveoli were statistically significant $(\mathrm{p}<0.05)$.

\section{Discussion}

This study allows a comparison between simple morphometric parameters of lung PAS in two discrete conditions: naturally collapsed organs and inflated lungs up to inspiratory reserve volume. The reduction of porosity of the subpleural layer in the collapsed lung is associated with the production of confluent B-lines and white lung, not associated to any specific histologic landmark or pathology. In naturally deflated lungs, lung density is close to $0.60 \mathrm{~g} / \mathrm{ml}$ and thus closer to the density of water and nonaerated tissues than to the density of a physiologically aerated lung $(0.15 \mathrm{~g} / \mathrm{ml})$.

Sonographic interstitial syndrome appears whenever the interaction of ultrasound pulses on the visceral pleu- 


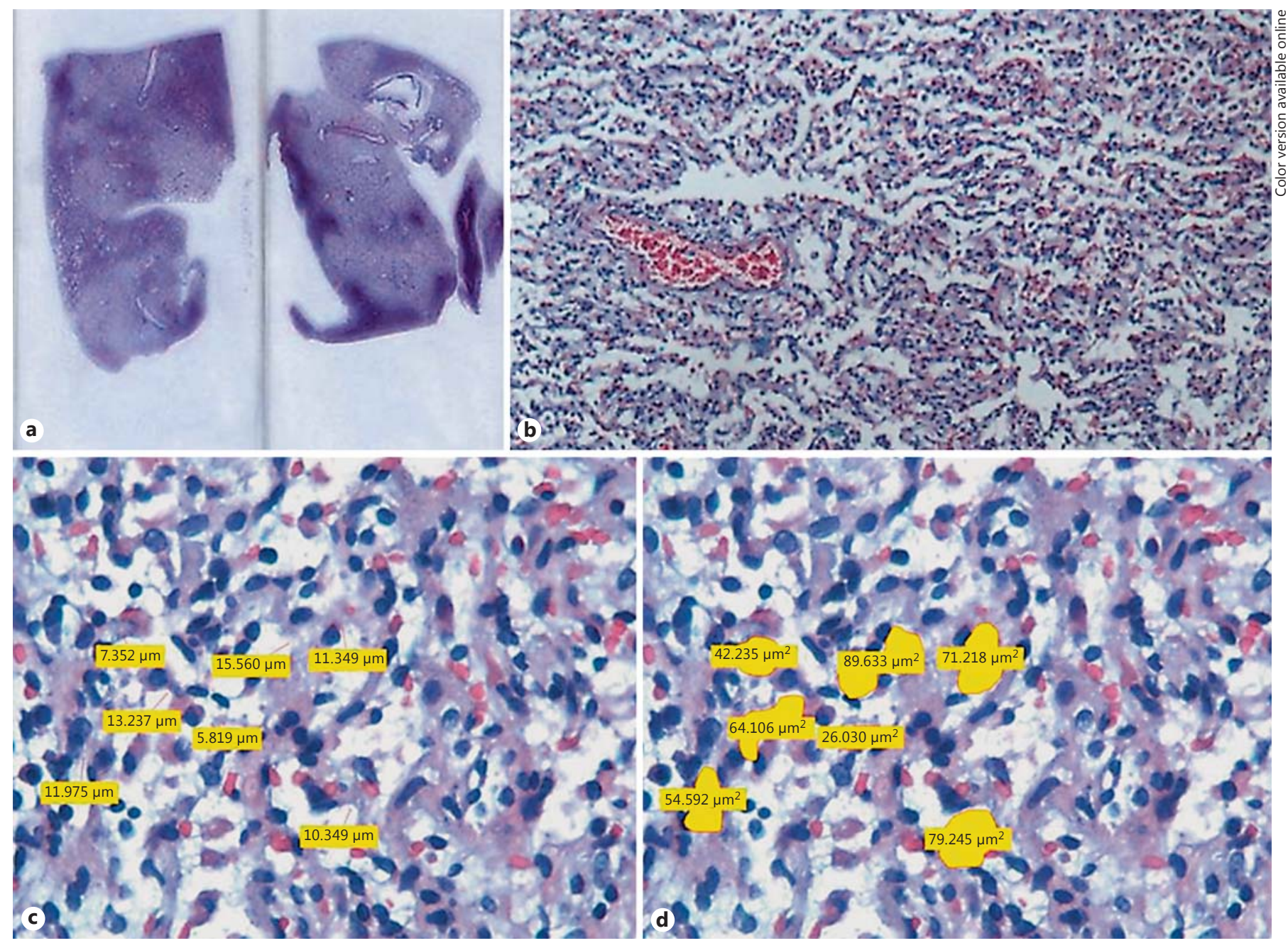

Fig. 3. Fixed collapsed lungs. a Anatomic image from 2 slides obtained from the blocks. b Histologic examination: diffusely compressed, closed and unfolded alveoli mixed with poorly aerated airspaces $(\times 10)$. Diameters $(\mathbf{c})$ and surface areas $(\mathbf{d})$ of 7 contiguous airspaces $(\times 40)$. The interstitial tissue is normal.

ral surface does not result in a specular reflection (mirror phenomenon) and acoustic events of scattering with or without wave interference take place in subpleural tissues, creating vertical artifacts and the white lung. These vertical artifacts indicate that the acoustic mirror 'breaks' and something changes on the lung surface $[20,21,26]$

Traditionally, B-lines have been attributed to discrete macroscopic or microscopic anatomic entities in the subpleural space. The importance of subpleural interlobular septa, or of thickening of subpleural connective tissue, has been highlighted by such terms as 'interstitial' or 'interstitial-alveolar syndrome', defining a pictorial ultrasound pattern of multiple ultrasound lung comets (Blines), which can be observed in pathologic lungs with a thickened interstitial space, as in the case of pulmonary edema, pulmonary fibrosis, interstitial inflammation and others $[3,5,7,11]$.

However, the possibility to create similar artifacts in synthetic foamy phantoms [20] or in bubble layers [26], their different shape, number and distribution relative to probe position or frequency, indicates the 'septal hypothesis' to be incomplete [27]. Moreover, the linear density (number/linear dimension) of artifacts similar to B-lines along an acoustic interface of a spongy phantom seems to correlate to its physical density [20].

Our experience has shown how sonographic interstitial syndrome can be produced not only by a pathological event, but also by a pure increase in density of the super- 


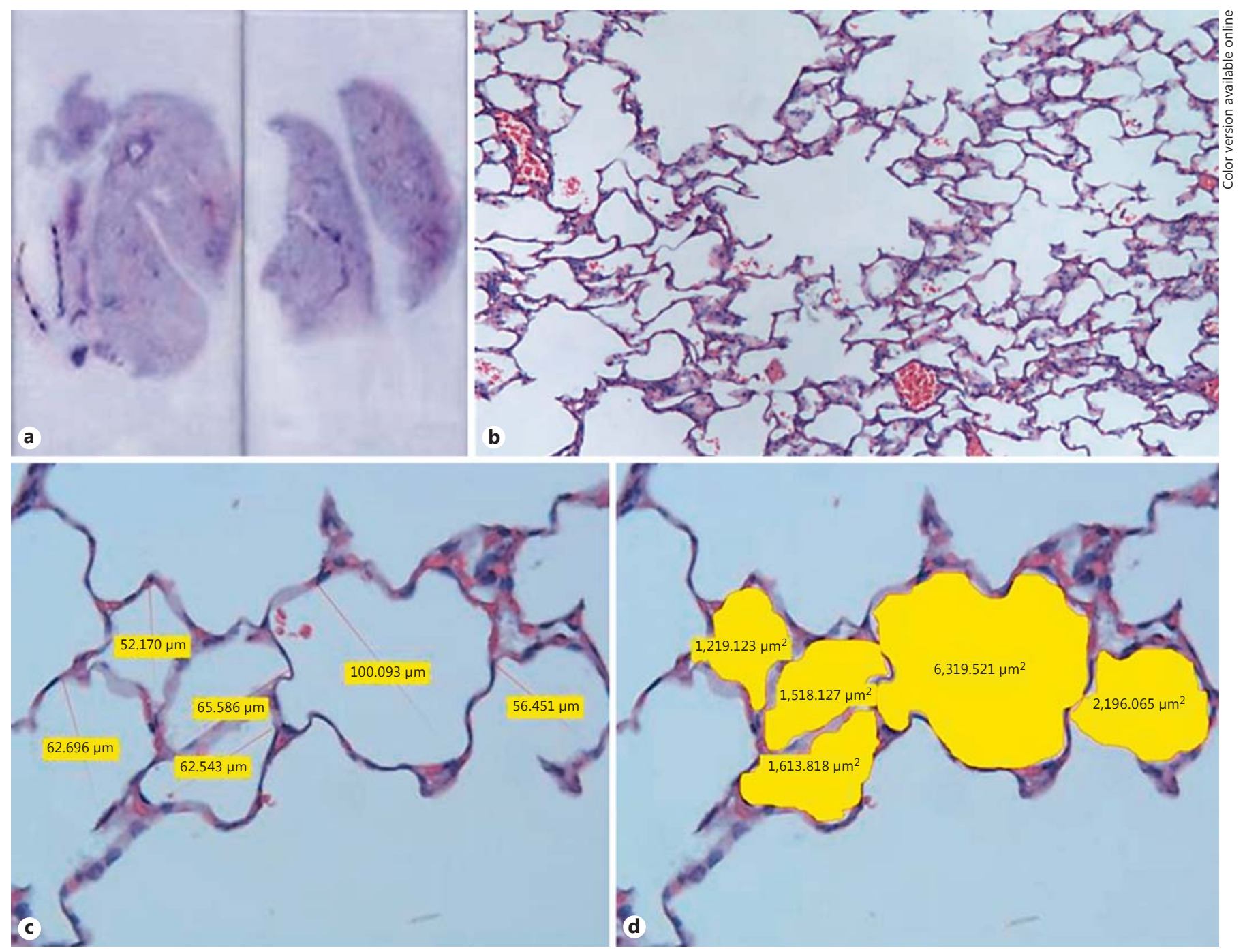

Fig. 4. Fixed inflated lungs. a Anatomic image from 2 slides obtained from the blocks. b Histologic examination: overinflated and opened alveoli and expanded alveolar sacs $(\times 10)$. Diameters $(\mathbf{c})$ and surface areas (d) of 6 contiguous air spaces $(\times 40)$.

ficial lung parenchyma obtained by lung deflation [21]. B-lines and white lung can be obtained either by reducing the volume of a healthy lung or by increasing its mass, as in inflammatory disease, pulmonary edema and interstitial lung pathology, whenever the ratio between the amount of water/cells/tissues and air is increased, independent of any specific anatomic landmark.

The presence of $\mathrm{B}$-lines and white lung in pulmonary areas of a CT ground glass pattern is in line with this hypothesis, if we consider the nature and the definition of 'preconsolidating' densities seen on CT named ground glass [28]. According to the results obtained, we are able to support the fact that a preconsolidating state of the cor- tical lung does not allow the ultrasound beam (in the frequency range of a commercial $9-\mathrm{MHz}$ probe) to penetrate the whole tissue volume to build clear anatomic pictures (as in pneumonia), and also not be specularly reflected producing A-lines (as in normal lung). Whenever the tissue/air ratio density is close to $0.60 \mathrm{~g} / \mathrm{ml}$, the ultrasound wavelength of a commercial linear probe operating at a nominal frequency of $9 \mathrm{MHz}$ must interact with acoustically active surface targets to dramatically change the visual ultrasound pattern returned, and thus erase A-lines.

On the pleural surface of healthy lungs the only possible acoustic targets are solid 'receptive microholes' surrounded by alveolar air of residual PAS. Inside these 

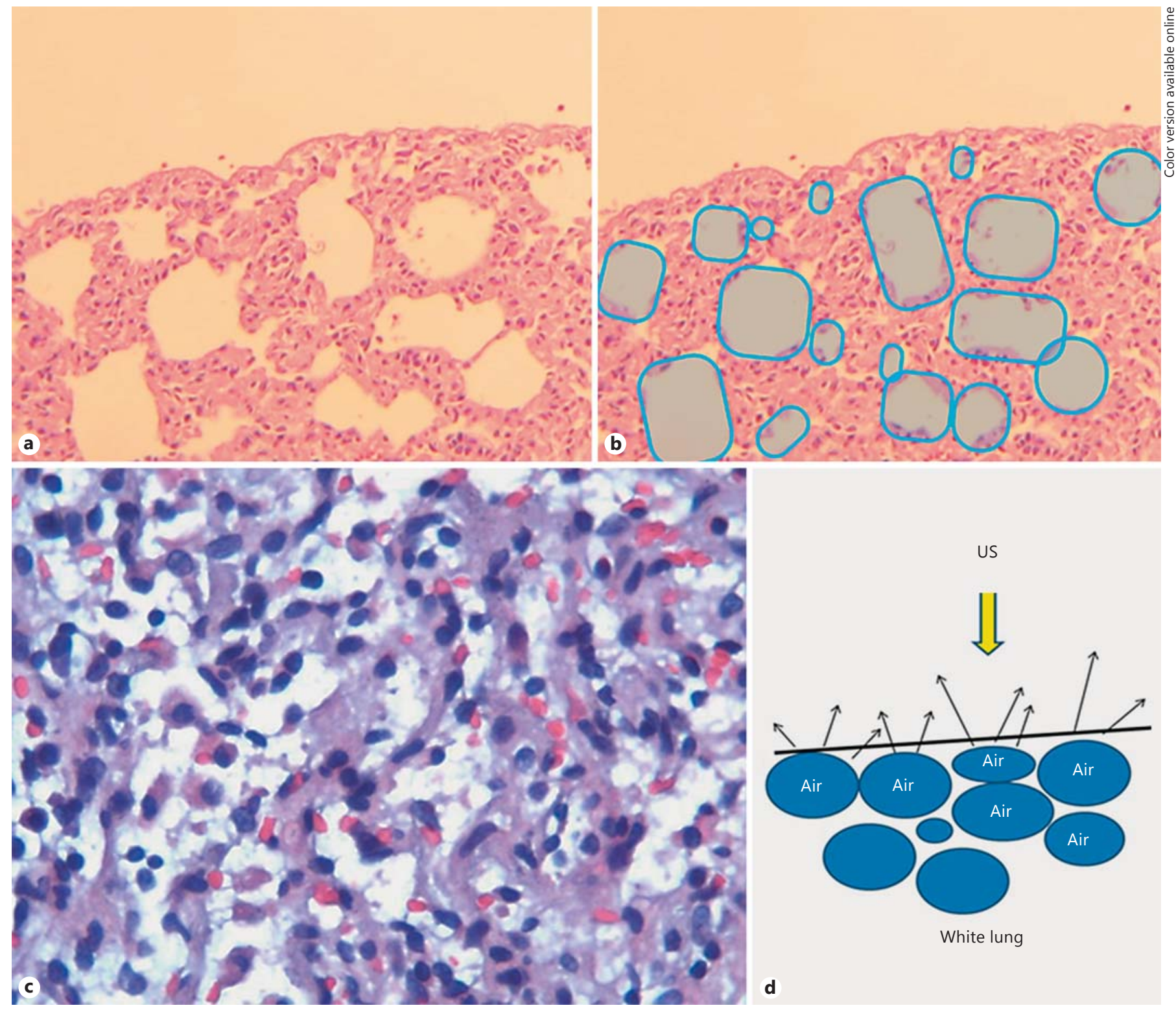

Fig. 5. Study of PAS in collapsed lungs. a-c Closed and unfolded PAS modifies airspace geometry with bullous disposition and less porosity of the solid open network of the frothy substrate. $\mathbf{d}$ The ultrasound (US) beam, depending on its wavelength, faces acoustic microholes with complex phenomena of backscattering and interference, resulting in vertical artifacts.

holes, poorly understood, complex acoustic events of scattering and/or wave interference change the returning signal (in the time and/or frequency domain), and this is interpreted by the ultrasound machine with artifact formation (B-line or white lung) [29].

These 'acoustic microholes' are related to the spatial remodeling of airspaces of the collapsed lung determining a critical air solid ratio or the 'breakage of the mirror'.
According to this view, a fully expressed ultrasound interstitial syndrome would be the hallmark of a preconsolidated lung (fig. 5, 6).

When studying lungs in in vivo and ex vivo conditions, other pertinent aspects deserve to be discussed. First, we have seen how the spongy network of the lung has elastic and dynamic characteristics so that airspace remodeling and, therefore, lung porosity and density, 

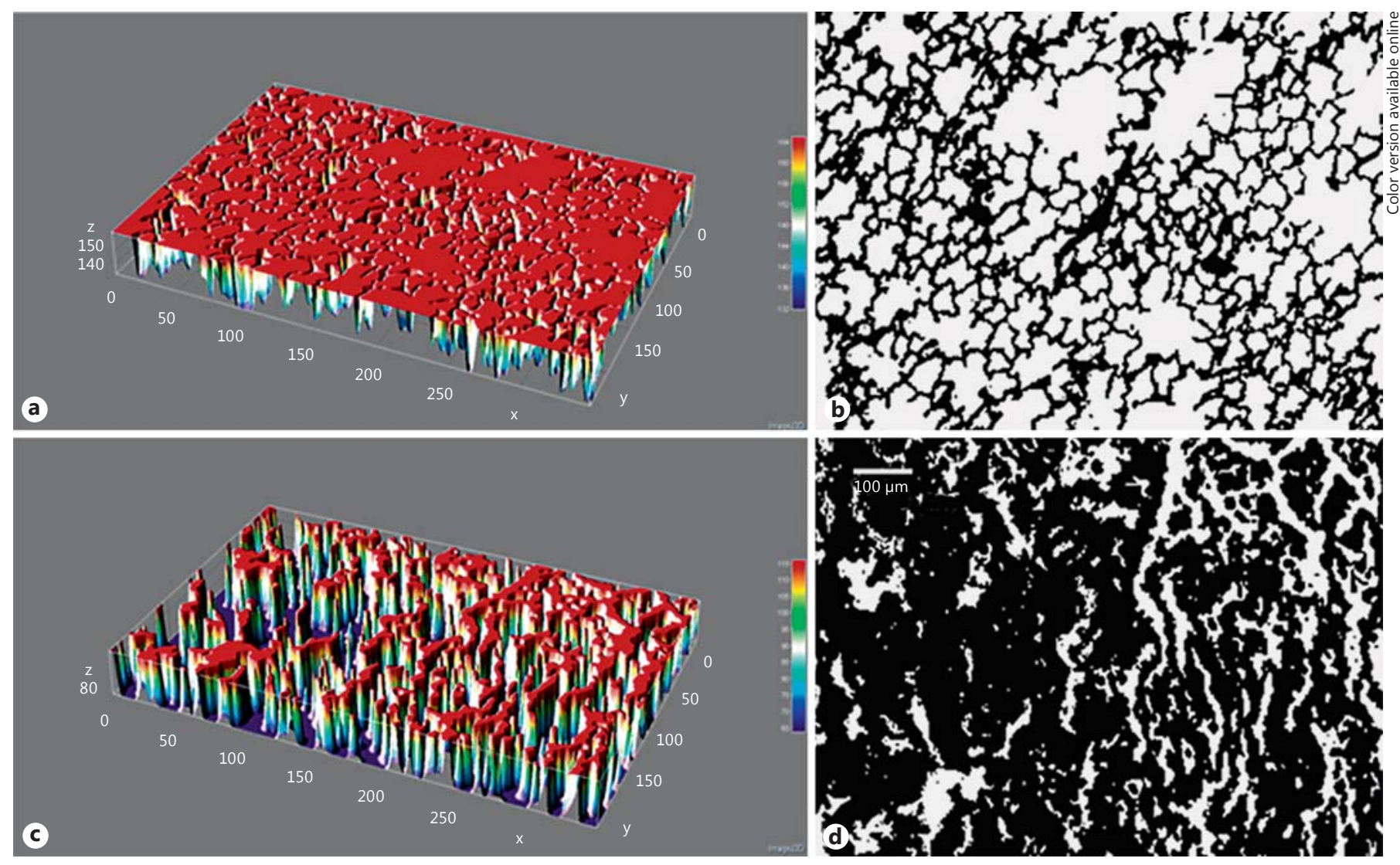

Fig. 6. a, c Three-dimensional surface digital reconstruction of a histological $40 \times$ field (red = empty; color refers to online version only) using the thermal LUT option (Image J): inflated lung (a) and deflated lung (c). b, d Related histological threshold $\times 40$ images: the surface of an inflated lung (b) is clearly more porous than the surface of a deflated lung (d). According to the literature, over a density range between 0.15 and $0.62 \mathrm{~g} / \mathrm{ml}$, the lung becomes anisotropically more dense and less porous. In $\mathbf{a}$ and $\mathbf{b}$, superficial acoustic holes are significantly larger than in $\mathbf{c}$ and $\mathbf{d}$, permitting the permeation of acoustic waves (in a characteristic range of holerelated frequencies) able to generate backscatter rather than specular reflection. changes with inflation or deflation, PAS recruitment and derecruitment [23], as well as with primary thickening of the interalveolar septa. PAS (respiratory bronchioles, alveolar ducts and alveolar sacs) are covered by a continuous thin liquid layer bearing a film of surfactant. The theory that this film arranges itself in bubbles that fill distal airspaces has already been presented by Scarpelli [30]. In this way, bubbles in PAS are organized to provide stability and to sustain aeration of lung parenchyma, and may be represented as an elastic, dynamic foam. These two components of the lungs' 'frothy' nature (solid open network and intra-alveolar bubbles) may explain its sonographic behavior, as has been reproduced in a real aqueous wet foam, whenever the superficial porosity and density change with pathological or functional variation of the air/tissue ratio takes place. In our previous experience, a wet foam is a strong producer of B-line-like artifacts [26].

Second, it has been suggested that the shape and the number of opened PAS may be modified as a function of lung volume. Mead et al. [31] described lung hysteresis relating lung expansion/deflation with a sequential opening and closing of units back in 1957 . This hypothesis was subsequently confirmed and sustained by other authors [32-34].

The concept of alveolar recruitment $[35,36]$ proposes that during inflation/deflation the lungs do not expand and retract isotropically, due to a sequential opening and closing of the PAS. In particular, during inflation there should be contemporaneously four possible ways in which PAS modify their shape and number: (1) opening of units (which explains the lower inflation point of the 


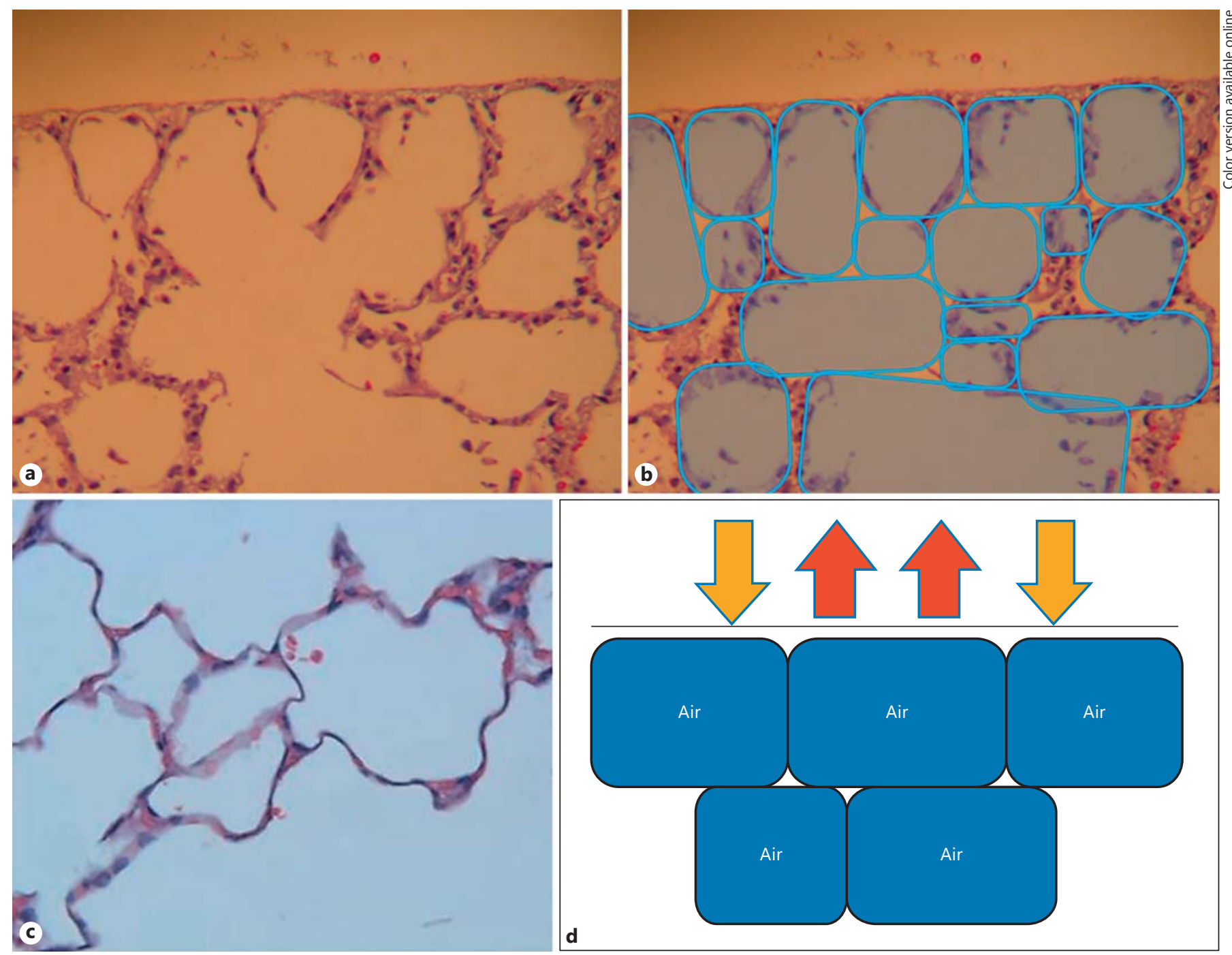

Fig. 7. Study of PAS in inflated lungs. Distended and opened airspaces dramatically change the airspace geometry, increasing porosity and impeding the ultrasound beam in recognizing acoustic microholes, determining a simple specular reflection.

pressure/volume curve), (2) change from dodecahedron to spherical shape, (3) balloon-like increase/reduction in alveolar size and (4) unfolding of 'collapsed' alveolar walls and septa. During deflation, there should be a point in which the opening zone ends and a sequential closing of PAS takes place [37, 38].

Our hypothesis is in accord with these physiological considerations and in particular with the evidence that the number of PAS decreases with lung volume decrease (derectruitment) [39]. Acoustic microholes consist in both closed PAS and unfolded alveolar walls and septa, as well as in thickened pathologic interstitium. These fea- tures are very evident in our histological images (fig. 1, 2, $5-7)$.

In pathologic lungs, PAS geometry and density may largely be changed through derecruitment and folding of airspaces, but other structural changes operate to produce the hyperdense preconsolidated tissue that generates artifacts. For example, in fibrotic lungs, thickening of the interstitium and the complete upheaval of PAS takes place. In edematous lungs, liquid first accumulates in the perivascular and interstitial compartment, thickening it, then enters the airspaces determining a transition from the air-filled to the fluid-filled state so that intra-alveolar 
air bubbles become free spherical bubbles [40]. In this case PAS geometry of the 'pulmonary foam' completely changes, in terms of reduced porosity, towards a wet foam [26]. On the other hand, when a lung is inflated, its porosity and airspace geometry dramatically changes and its acoustic behavior becomes that of a dry foam able to specularly reflect the beam [41] (fig. 6, 7).

Histological features of the lung and corresponding echographic patterns fully support this theory. Visual patterns of sonographic interstitial syndrome may therefore be interpreted as indirect 'estimators' of porosity of the superficial lung parenchyma or, in other words, a sort of 'visual' scan of the 'acoustic roughness' of the pleural plane. Only when lung porosity shows features similar to that of 'solid tissues' in terms of acoustic impedance can the ultrasound beam go beyond the pleural plane, producing consolidation images.

\section{Conclusions}

This study is a preliminary experience, but it highlights a fundamental topic. Vertical artifacts are related to the wavelength used and to the possibility to see (or better 'to listen to') acoustic microholes generated by PAS disposition. The lung is a fractal and a frothy substrate for ultrasounds and, in our opinion, it is not exactly the density of the lungs in terms of water/tissues/air that deserves to be focused on, but the porosity in terms of shape and disposition of air. Altered density of peripheral lung is an epiphenomenon of altered PAS geometry. This explains why similar artifactual phenomena could be seen in other contests (abdomen, phantoms, etc.) and why vertical artifacts seem to differ from one another in different lung pathologies (PAS disposition is different between cardiogenic edema, pneumonic edema, pulmonary fibrosis) and when using different probes and wavelengths.

To date, generation of sonographic interstitial syndrome is not completely clear and the visual representation of lung artifacts (B-lines and white lung) by the machine is too simplistic. Commercial echographic equipment has been optimized to work with a low acoustic impedance mismatch and in general does not provide primitive radiofrequency signals or the possibility to tune key acquisition parameters such as ultrasound carrier frequency and pulse bandwidth, which would be needed for future applications and to better discriminate subpleural interstitial pathology [42]. This study adds to other works $[20,21,26,27,43,44]$ in changing the interpreting theories of sonographic interstitial syndrome and allowing a better understanding and classification of physiological and pathological lung echographic patterns in terms of artifacts.

\section{Acknowledgements}

We are indebted to Marcello Demi (Department of Medical Image Processing, Fondazione Toscana Gabriele Monasterio, Pisa, Italy) and Libertario Demi (Laboratory of Biomedical Diagnostics, Eindhoven University of Technology, Eindhoven, The Netherlands) for their physical and technical help.

\section{Financial Disclosure and Conflicts of Interest}

No conflict of interest has to be reported in relation to this work.

\section{References}

\footnotetext{
1 Zanforlin A, Giannuzzi R, Nardini S, Testa A, Soldati G, Copetti R, Marchetti G, Valente S, Inchingolo R, Smargiassi A: The role of chest ultrasonography in the management of respiratory diseases: document I. Multidiscip Respir Med 2013;8:54.

2 Smargiassi A, Inchingolo R, Soldati G, Copetti R, Marchetti G, Zanforlin A, Giannuzzi R, Testa A, Nardini S, Valente S: The role of chest ultrasonography in the management of respiratory diseases: document II. Multidiscip Respir Med 2013;8:55.

-3 Koegelenberg CF, von Groote-Bidlingmaier F, Bolliger CT: Transthoracic ultrasonography for the respiratory physician. Respiration 2012;84:337-350.
}

\footnotetext{
4 Volpicelli G, Elbarbary M, Blaivas M, Lichtenstein DA, Mathis G, Kirkpatrick AW, Melniker L, Gargani L, Noble VE, Via G, Dean A, Tsung JW, Soldati G, Copetti R, Bouhemad B, Reissig A, Agricola E, Rouby JJ, Arbelot C, Liteplo A, Sargsyan A, Silva F, Hoppmann R, Breitkreutz R, Seibel A, Neri L, Storti E, Petrovic $\mathrm{T}$, International Liaison Committee on Lung Ultrasound (ILC-LUS) for International Consensus Conference on Lung Ultrasound (ICC-LUS): International evidencebased recommendations for point-of-care lung ultrasound. Intensive Care Med 2012;38: 577-591.
}

Lung Ultrasonography and Estimation of Porosity and Airspace Geometry 
5 Reissig A, Copetti R: Lung ultrasound in community-acquired pneumonia and in interstitial lung diseases. Respiration 2014;87:179189.

6 Stigt JA, Groen HJ: Percutaneous ultrasonography as imaging modality and sampling guide for pulmonologists. Respiration 2014; 87:441-451.

7 Kreuter M, Mathis G: Emergency ultrasound of the chest. Respiration 2014;87:89-97.

8 Dunn F, Fry WJ: Ultrasonic absorption and reflection by lung tissue. Phys Med Biol 1961; 5:401-410.

-9 Reissig A, Gramegna A, Aliberti S: The role of lung ultrasound in the diagnosis and followup of community-acquired pneumonia. Eur J Intern Med 2012;23:391-397.

10 Reissig A, Copetti R, Mathis G, Mempel C, Schuler A, Zechner P, Aliberti S, Neumann R, Kroegel C, Hoyer H: Lung ultrasound in the diagnosis and follow-up of communityacquired pneumonia: a prospective, multicenter, diagnostic accuracy study. Chest 2012; 142:965-972.

11 Lichtenstein D, Mezière G, Seitz J: The dynamic air bronchogram: a lung ultrasound sign of alveolar consolidation ruling out atelectasis. Chest 2009;135:1421-1425.

12 Koenig SJ, Narasimhan M, Mayo PH: Thoracic ultrasonography for the pulmonary specialist. Chest 2011;140:1332-1341.

13 Copetti R, Soldati G, Copetti P: Chest sonography: a useful tool to differentiate acute cardiogenic pulmonary edema from acute respiratory distress syndrome. Cardiovasc Ultrasound 2008;29:6-16.

14 Avruch L, Cooperberg PL: The ring-down artifact. J Ultrasound Med 1985;4:21-28.

15 Kohzaki S, Tsurusaki K, Uetani M, Nakanishi $\mathrm{K}$, Hayashi K: The aurora sign: an ultrasonographic sign suggesting parenchymal lung disease. Br J Radiol 2003;76:437-443.

16 Lichtenstein D, Mézière G, Biderman P, Gepner A, Barré O: The comet-tail artifact: an ultrasound sign of alveolar-interstitial syndrome. Am J Respir Crit Care Med 1997;156: 1640-1646.

$\$ 17$ Miller DL: Induction of pulmonary hemorrhage in rats during diagnostic ultrasound. Ultrasound Med Biol 2012;38:1476-1482.
18 Dunn F: Attenuation and speed of ultrasound in lung: dependence upon frequency and inflation. J Acoust Soc Am 1986;80:1248-1250.

-19 O’Brien WD, Kramer JM, Waldrop TG, Frizzel LA, Miller RJ, Blue JP, Zachary JF: Ultrasound-induced lung hemorrhage: role of acoustic boundary conditions at the pleural surface. J Acous Soc Am 2002;111:1102-1109.

20 Soldati G, Giunta V, Sher S, Melosi F, Dini C: 'Synthetic' comets: a new look at lung sonography. Ultrasound Med Biol 2011;37:17621770.

21 Soldati G, Inchingolo R, Smargiassi A, Sher S, Nenna R, Inchingolo CD, Valente S: Ex vivo lung sonography: morphologic-ultrasound relationship. Ultrasound Med Biol 2012;38: 1169-1179.

2 Yan X, Polo Carbayo JJ, Weibel ER, Hsia CC Variation of lung volume after fixation when measured by immersion or Cavalieri method. Am J Physiol Lung Cell Mol Physiol 2003; 284:L242-L245.

23 Escolar JD, Escolar MA, Blasco J, Ros LH: Airway and lung parenchyma during the respiratory cycle. Histol Histopathol 2007;22:43-49.

24 Schibler A, Henning R: Measurement of functional residual capacity in rabbits and children using an ultrasonic flow meter. Pediatr Res 2001;49:581-588.

25 Stahl WR: Scaling of respiratory variables in mammals. J Appl Physiol 1967;22:453-460.

26 Soldati G, Copetti R, Sher S: Sonographic interstitial syndrome: the sound of lung water. J Ultrasound Med 2009;28:163-174.

27 Woitczak JA, Wood RW: High frequency ultrasound in ex vivo animal lung in pulmonary edema. J Anesthesiol Clin Sci 2013;2:21.

28 Miller WT, Shah RM: Isolated diffuse ground glass opacity in thoracic CT: causes and clinical presentations. AJR Am J Roentgenol 2005; 184:613-622.

29 Mikhak Z, Pedersen PC: Acoustic attenuation properties of the lung: an open question. Ultrasound Med Biol 2002;28:1209-1216.

30 Scarpelli EM: The alveolar surface network: a new anatomy and its physiological significance. Anat Rec 1998;251:491-527.

31 Mead J, Whittenberger JL, Radford EP Jr: Surface tension as a factor in pulmonary volumepressure hysteresis. J App Physiol 1957;10: 191-196.
32 Frazer DG, Weber KC, Franz GN: Evidence of sequential opening and closing of lung units during inflation of excised rat lungs. Respir Physiol 1985;61:277-288.

33 Cheng W, DeLong DS, Franz GN, Petsonk EL, Frazer DG: Contribution of opening and closing of units to lung hysteresis. Respir Physiol 1995; 102:205-215.

34 Gil J, Weibel ER: Morphological study of pressure volume hysteresis in rat lungs fixed by vascular perfusion. Respir Physiol 1972;15: 190-213.

35 Smaldone GC, Mitzner W, Itoh H: Role of alveolar recruitment in lung inflation: influence on pressure-volume hysteresis. J Appl Physiol Repirat Environ Exercise Physiol 1983;55: 1321-1332.

36 Carney DE, Brendenberg CE, Shiller HJ, Picone AL, McCann UG, Gatto LA, Bailey G, Fillimger M, Nieman GF: The mechanism of lung volume change during mechanical Ventilation. Am J Respir Crit Care Med 1999;160: 1697-1702.

37 Escolar JD, Escolar A: Lung hysteresis: a morphological view. Histol Histopathol 2004;19: 159-166.

38 Gil J, Bachofen H, Gehr P, Weibel ER: Alveolar volume-surface area relation in air- and saline-filled lungs fixed by vascular perfusion. J Appl Physiol 1979;47:990-1001.

39 Escolar JD, Escolar MA, Guzman J, Roques M: Pressure volume curve and alveolar recruitment: a morphometric model of the respiratory cycle. Histol Histopathol 2002;17: 383-392.

-40 Wilson TA, Anafi RC, Hubmayr RD: Mechanics of edematous lungs. J Appl Phisiol 2001;90:2088-2093.

41 Weaire D, Hutzler S: The Physics of Foam. Oxford, Oxford University Press, 1999.

42 Demi L, Demi M, Smargiassi A, Inchingolo R, Faita F, Soldati G: Ultrasonography in lung pathologies: new perspectives. Multidiscip Respir Med 2014;9:27.

43 Soldati G, Sher S, Testa A: Lung and ultrasound: time to 'reflect'. Eur Rev Med Pharmacol Sci 2011;15:223-227.

44 Volpicelli G: Lung sonography. J Ultrasound Med 2013;32:165-171. 